\title{
Predictive MGMT status in a homogeneous cohort of IDH wildtype glioblastoma patients
}

Josefine Radke ${ }^{1,2,7^{*}+}$ (D) Arend Koch ${ }^{1,2,7 \dagger}$, Fabienne Pritsch ${ }^{1,2}$, Elisa Schumann ${ }^{1,2}$, Martin Misch $^{3}$, Claudia Hempt $^{3}$, Klaus Lenz ${ }^{4}$, Franziska Löbel ${ }^{3}$, Fabienne Paschereit ${ }^{3}$, Frank L. Heppner ${ }^{1,2,5,8}$, Peter Vajkoczy ${ }^{3}$, Randi Koll1,2 and Julia Onken ${ }^{3,6^{*}}$

\begin{abstract}
Methylation of the O(6)-Methylguanine-DNA methyltransferase (MGMT) promoter is predictive for treatment response in glioblastoma patients. However, precise predictive cutoff values to distinguish "MGMT methylated" from "MGMT unmethylated" patients remain highly debated in terms of pyrosequencing (PSQ) analysis. We retrospectively analyzed a clinically and molecularly very well-characterized cohort of $111 \mathrm{IDH}$ wildtype glioblastoma patients, who underwent gross total tumor resection and received standard Stupp treatment. Detailed clinical parameters were obtained. Predictive cutoff values for MGMT promoter methylation were determined using ROC curve analysis and survival curve comparison using Log-rank (Mantel-Cox) test. MGMT status was analyzed using pyrosequencing (PSQ), semiquantitative methylation specific PCR (sqMSP) and direct bisulfite sequencing (dBiSeq). Highly methylated (> 20\%) MGMT correlated with significantly improved progression-free survival (PFS) and overall survival (OS) in our cohort. Median PFS was 7.2 months in the unmethylated group (UM, <10\% mean methylation), 10.4 months in the low methylated group ( $L M, 10-20 \%$ mean methylation) and 19.83 months in the highly methylated group (HM, $>20 \%$ mean methylation). Median OS was 13.4 months for UM, 17.9 months for LM and 29.93 months for HM. Within the LM group, correlation of PSQ and sqMSP or dBiSeq was only conclusive in $51.5 \%$ of our cases. ROC curve analysis revealed superior test precision for survival if additional sqMSP results were considered (AUC $=0.76$ ) compared to PSQ (cutoff $10 \%$ ) alone (AUC $=0.67$ ). We therefore challenge the widely used, strict PSQ cutoff at $10 \%$ which might not fully reflect the clinical response to alkylating agents and suggest applying a second method for MGMT testing (e.g. MSP) to confirm PSQ results for patients with LM MGMT levels if therapeutically relevant.
\end{abstract}

Keywords: Glioblastoma, Temozolomide (TMZ), O(6)-Methylguanine-DNA methyltransferase (MGMT), Methylation specific PCR (MSP), Pyrosequencing (PSQ), IDH (isocitrate dehydrogenase)

\footnotetext{
* Correspondence: josefine.radke@charite.de; julia.onken@charite.de

${ }^{\dagger}$ Josefine Radke and Arend Koch contributed equally to this work.

${ }^{1}$ Department of Neuropathology, Charité - Universitätsmedizin Berlin,

corporate member of Freie Universität Berlin, Humboldt-Universität zu Berlin, and Berlin Institute of Health, Charitéplatz 1 (Virchowweg 15), 10117 Berlin, Germany

${ }^{3}$ Department of Neurosurgery, Charité - Universitätsmedizin Berlin, corporate member of Freie Universität Berlin, Humboldt-Universität zu Berlin, and Berlin Institute of Health, Charitéplatz 1, 10117 Berlin, Germany

Full list of author information is available at the end of the article
}

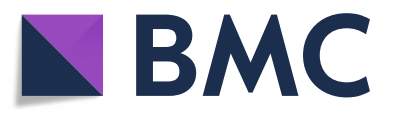

(c) The Author(s). 2019 Open Access This article is distributed under the terms of the Creative Commons Attribution 4.0 International License (http://creativecommons.org/licenses/by/4.0/), which permits unrestricted use, distribution, and reproduction in any medium, provided you give appropriate credit to the original author(s) and the source, provide a link to the Creative Commons license, and indicate if changes were made. The Creative Commons Public Domain Dedication waiver (http://creativecommons.org/publicdomain/zero/1.0/) applies to the data made available in this article, unless otherwise stated. 


\section{Introduction}

Glioblastoma (GBM) is the most common and most aggressive primary brain tumor. The histological examination of neurosurgical tumor specimens as well as the immmunohistochemical or molecular determination of the $I D H 1 / 2$ status remain the gold standard for diagnosis of GBM [13]. Despite aggressive therapy, the survival of patients with GBM is approximately 15-17 months [21]. The current standard GBM therapy usually consists of neurosurgical resection, radiotherapy and additional chemotherapy with temozolomide (TMZ), an alkylating agent. But, chemosensitivity to TMZ strongly depends on epigenetic silencing by methylation of the $\mathrm{O}(6)$ Methylguanine-DNA methyltransferase (MGMT) promoter [15]. Different randomized trials have shown that methylation of the MGMT promoter in GBM patients is associated with significantly higher survival rates if treated with radiotherapy and TMZ [4]. At the stage of recurrent disease, a TMZ rechallenge seems only reasonable in patients with clear methylation of the MGMT promoter based on the results of the DIRECTOR trial [24]. Recent data from the NOA-09 trial showed that newly diagnosed GBM patients with methylated MGMT promoter might benefit from a more intense first-line treatment regimen with $\mathrm{CCNU}$ in combination with TMZ [8], accepting an increased toxicity for an improved prognosis. These trials emphasize the importance of reliable MGMT status assessment and the need for predictive cutoff levels for clinical decision-making.

The methylation status of the MGMT promoter is widely determined by quantitative pyrosequencing (PSQ) $[12,28]$. PSQ analysis uses a defined cutoff value to classify cases as "methylated" or "unmethylated" [1]. In many neurooncological centers, the biological cutoff is $10 \%$ [27]. However, a very strict cutoff value might not fully reflect the clinical response to TMZ therapy. Various previous studies that focused on the technical assessment of the MGMT status have suggested higher predictive cutoff levels above $10 \%$ [14, 17, 18].

Here, we aimed to determine a predictive cutoff level for clinical decision-making on the basis of a welldefined patient cohort of $111 \mathrm{IDH}$ wildtype GBM patients. Three methylation groups were identified, which showed a very distinct clinical course in terms of PFS and OS: unmethylated 0-9\% (UM), low methylated 10$20 \%$ (LM), and highly methylated > 20\% (HM).

\section{Methods and Material}

\section{Tissue samples, clinical and patient data}

Two hundred ninety patients with newly diagnosed, previously untreated GBM (WHO grade IV) patients have been diagnosed between 2010 and 2015 at the Departments of Neurosurgery and Neuropathology, Charité Berlin, Germany. GBM diagnosis was confirmed by at least two experienced neuropathologists after surgical resection or stereotactic biopsy. According to the current WHO classification of CNS tumors [13], IDH mutation status was determined by IDH1 R132H immunohistochemistry (IHC) and bidirectional Sanger sequencing of exon 4 of the IDH1 as well as IDH2 gene for all GBM patients younger than 55 years [13]. Gliosarcoma, epithelioid glioblastoma, giant cell glioblastoma and $I D H$ mutant tumors were excluded. The following clinical data were assessed: age at diagnosis, Karnofsky performance status (KPS), tumor localization, extent of resection and residual tumor volume, type and timing of adjuvant therapy, second-line therapy at recurrence, follow-up time, progression-free (PFS) and overall survival (OS) in months. The extent of tumor resection was determined by measuring the contrast-enhancing tumor volume in $\mathrm{mm}^{3}$ on $\mathrm{T} 1$-subtraction MRI imaging preand 48 hours postoperatively using the Brainlab iMRI software (Brainlab AG, Munich, Germany). Gross total resection (GTR) was defined as residual tumor volume < $2 \%$ [22]. PFS was assessed according to RANO criteria [25]. We identified $205 \mathrm{IDH}$ wildtype GBM patients who matched the criteria mentioned above. Three long-term survivors (LTS; OS > 5 years) were identified in our cohort. For two LTS cases, DNA was sufficient to perform a genome-wide methylation analysis (EPIC array) which confirmed the diagnosis of GBM, IDH wildtype (Additional file 1: Figure S3 and Figure S4).

\section{Ethical statement}

This study was conducted according to the ethical principles of medical research involving human subjects according to the Declaration of Helsinki. The clinical data were assessed and anonymized for patients' confidentiality. Ethical approval (EA2/064/17) was granted by the institutional ethics board of the Charité Ethics Committee.

\section{DNA extraction, bisulfite treatment and analysis of MGMT promoter methylation status in tumor samples}

Areas of high tumor cell content $(\geq 80 \%)$ were chosen and macro-dissected for further analysis (Additional file 1: Figures S1a, dashed line; 1 b). Genomic DNA was extracted from formalin-fixed and paraffin embedded (FFPE) samples using the Qiagen DNeasy blood and tissue DNA extraction kit according to the manufacturer's protocol (Qiagen, Hilden, Germany). The DNA was sodium bisulfite-modified using the EZ DNA MethylationGold $^{\text {ts }}$ Kit (Zymo Research, Irvine, CA).

Pyrosequencing (PSQ) Quantitative methylation analyzes were performed using the PyroMark Q24 MGMT kit (Qiagen, Hilden, Germany) and an automated PyroMark Q24 System (Qiagen, Hilden, Germany) following the manufacturer's instructions. Data was analyzed with 
the PyroMark Q24 Software 2.0 (Qiagen, Hilden, Germany). The percentage of methylated alleles was calculated as the mean value of the methylation percentage obtained. The cutoff value $\geq 10 \%$ was defined to classify MGMT methylated vs. unmethylated cases, which is commonly used and has been validated for routine clinical diagnostics [27]. Standardized positive and negative controls were included in every PSQ run. The PSQ results were evaluated by at least two experienced neuropathologists.

Semi-quantitative methylation-specific PCR (sqMSP) sqMSP was performed with primers specific for either "methylated" or "unmethylated" DNA as previously described [5]. Original MSP PCR gels are shown in Additional file 1: Figure S2. Primers and PCR programs are listed in the methods and material section of Additional file 1. Semi-quantitative analysis of the optical band intensity (I) was performed using ImageJ (National Institutes of Health, Bethesda, USA). The following equation was used:

Band intensity methylated in $\%$

$$
=\left(\left(\mathrm{I}_{\text {meth }}+\mathrm{I}_{\text {unmeth }}\right) / \mathrm{I}_{\text {meth }}\right) \times 100
$$

\section{Band intensity unmethylated in $\%$}

$$
=\left(\left(\mathrm{I}_{\text {meth }}+\mathrm{I}_{\text {unmeth }}\right) / \mathrm{I}_{\text {unmeth }}\right) \times 100
$$

\section{Direct Bisulfite Sequencing (dBiSeq)}

dBiseq was carried out as previously described [16] with minor adaptations. Primers and PCR program are listed in the methods and material section of Additional file 1. Sequencing was performed at Eurofins Genomics, Ebersberg, Germany. Sequenced samples were returned as .ab1 files, which were then analyzed using Chromas [9] (software program for PC, available at http://www.technelysium.com.au/chromas.html).

\section{Analysis of MGMT promoter methylation status in positive and negative controls}

Both, positive and negative controls (listed in Additional file 1: Table S1) were assessed by PSQ, sqMSP, and dBiseq. Samples of non-neoplastic brain tissue and one samples with genomic DNA extracted from whole peripheral blood served as negative controls. The primary cell line SF126 and 7 tumor samples with clear MGMT promoter methylation levels $>30 \%$ were used as positive controls.

\section{Genome-wide DNA methylation analysis}

DNA methylation signature analysis was performed using the Illumina Infinium Methylation EPIC array as previously described [2].

\section{$I D H 1$ and IDH2 Sanger sequencing}

Bidirectional Sanger sequencing of exon 4 of $I D H 1$ and IDH2 was performed in IDH R132H IHC-negative or -equivocal cases in all patients $<55$ years of age. PCR primers for the genomic regions corresponding to $I D H 1$ exon 4 (codon R132) and $I D H 2$ exon 4 (codon R172) and the flanking intronic sequences are displayed in the methods and material section of Additional file1. Sequencing was performed at Eurofins Genomics, Ebersberg, Germany.

\section{Immunohistochemical procedures}

Immunohistochemical stainings were performed on a Benchmark XT autostainer (Ventana Medical Systems, Tuscon, AZ, USA) with standard antigen retrieval methods (CC1 buffer, pH8.0, Ventana Medical Systems, Tuscon, AZ, USA) using 4- $\mu \mathrm{m}$-thick, FFPE tissue sections (Additional file 1: Figures S1 c-f). The following primary antibodies were used: polyclonal rabbit anti-GFAP (1:2000, Dako), monoclonal mouse anti-MIB1 (Ki-67, 1:100, clone M7240, Dako), polyconal rabbit anti-ATRX (1:200, Sigma), mouse monoclonal anti-IDH1 R132H (1:20, clone H09, Dianova). The iVIEW DAB Detection Kit (Ventana Medical Systems, Tuscon, AZ, USA) was used according to the manufacturer's instructions. Sections were counterstained with hematoxylin, dehydrated in graded alcohol and xylene, mounted and coverslipped. IHC stained sections were evaluated by two independent, experienced neuropathologists. When no agreement was reached, the sections were reviewed by our team of neuropathologists at our department (Charité) and further molecular diagnostics (e.g. $I D H 1 / I D H 2$ bidirectional Sanger sequencing, genome-wide DNA methylation analysis (EPIC analysis)) was performed.

\section{Statistical analysis}

Statistical analysis was performed in cooperation with the Charité's Institute for Biometrics and Clinical Epidemiology using GraphPad Prism 5 (GraphPad Software, La Jolla, CA, USA). Kaplan-Meier survival curves were obtained and differences in PFS and OS were tested for statistical significance using the log-rank test. Significance level was set at $p<0.05$.

ROC analysis was used for diagnostic test evaluation. The true positive rate (Sensitivity) was plotted as a function of the false positive rate (100-Specificity) for different cutoff points. The area under the ROC curve (AUC) measured the accuracy. An AUC of 1 represents a perfect test; 0.8-0.9 a good test, 0.7-0.8 a fair test, 0.6-0.7 a poor test, and an area of $\leq 0.5$ represents a worthless test.

\section{Results}

Study cohort

Heterogeneity of the patient cohort (e.g. in terms of the $I D H$ status) has been a major point of criticism in 
previous studies where the predictive mean MGMT promoter methylation cutoff had to be determined. Therefore, we selected a homogeneous group of $I D H$ wildtype GBM patients with KPS > 70\%, who received i) GTR of GBM manifestation, ii) Stupp regime within 4-6 weeks after initial surgery [20], and iii) completed Stupp regime after 6 cycles or until progression of disease, assessed according to the RANO criteria $(n=111)$. All clinical information is displayed in Table 1. GBM diagnosis was confirmed by at least two experienced neuropathologists using a standardized panel of conventional and immunohistochemical stainings (Additional file 1: Figures S1 a-f) . All cases were proven $I D H$ wildtype by bidirectional Sanger sequencing. Patients with $I D H 1$ (Additional file 1: Figures S1 g-j) and IDH2 (Additional file 1: Figure S5) mutant tumors were excluded.

Initially, Kaplan-Meier curves were generated for the following methylation groups (mean MGMT promoter

Table 1 Patients' characteristics of our study cohort

\begin{tabular}{|c|c|c|c|}
\hline \multicolumn{2}{|l|}{ Study cohort $n=111$} & \multirow{2}{*}{$\begin{array}{l}\mathrm{n} \\
48\end{array}$} & \multirow{2}{*}{$\frac{\%}{43}$} \\
\hline Gender & Female & & \\
\hline & Male & 63 & 57 \\
\hline \multirow[t]{3}{*}{ Age in years } & Mean & 58.9 & - \\
\hline & Median & 61.2 & - \\
\hline & Range & $18-85.4$ & - \\
\hline \multirow[t]{2}{*}{ MGMT } & Meth. (mean $\geq 10 \%$ ) & 56 & 51 \\
\hline & Unmeth. (mean < 10\%) & 55 & 49 \\
\hline Toxicity during $1^{\text {st }}$ line therapy & CTG ${ }^{\circ} \|-I V$ & 4 & 4 \\
\hline \multirow[t]{7}{*}{$2^{\text {nd }}$ line therapy } & mTMZ & 38 & 34 \\
\hline & TMZ rechallenge & 7 & 6 \\
\hline & CCNU+Procarbazine & 4 & 4 \\
\hline & BEV & 3 & 3 \\
\hline & Re-irradiation & 4 & 4 \\
\hline & TTFields & 5 & 5 \\
\hline & Re-resection & 33 & 30 \\
\hline \multirow[t]{4}{*}{ Follow up in months } & Mean & 19.4 & - \\
\hline & Median & 15.4 & - \\
\hline & Range & $0.3-90$ & - \\
\hline & Lost to follow up & 2 & 2 \\
\hline \multirow[t]{3}{*}{ PFS in months } & Mean & 12 & - \\
\hline & Median & 7.8 & - \\
\hline & Range & $0.3-56$ & - \\
\hline \multirow[t]{3}{*}{ OS in months } & Mean & 19.8 & - \\
\hline & Median & 15.5 & - \\
\hline & Range & $0.5-90$ & - \\
\hline
\end{tabular}

BEV bevacizumab, CCNU lomustin, CTG common toxicity criteria, GTR gross total resection, $M G M T \mathrm{O}^{6}$-methylguanine-DNA-methyltransferase, meth methylated, $m T M Z$ metronomic temozolomide, OS overall survival, $P R$ partial resection, $T M Z$ temozolomide, TTFields tumor treating fields, unmeth unmethylated methylation): $0-9 \%, 10-20 \%, 21-30 \%, 31-40 \%$, and $>40 \%$ (Figure $1 \mathrm{a}, \mathrm{b})$. mPFS in months was 5.28 (0-9\%), 8.03 (1020\%), 22.4 (21-30\%), 16.13 (31-40\%), and 13.8 (> 40\%). mOS in months was 10.07 (0-9\%), 13.83 (10-20\%), 33.33 (21-30\%), 29.93 (31-40\%), and 19.43 (> 40\%). For PFS, Kaplan-Meier curves comparison revealed significant differences between the following groups: $0-9 \%$ vs. $10-20 \%$ (" $\mathrm{p}=0.0143$, HR 1.745 , CI 1.118 to 2.725$), 0-9 \%$ vs. $21-30 \%$ ${ }^{* * * * *} \mathrm{p}<0.0001$, HR 3.307 , CI1.885 to $5.800 ; 0-9 \%$ vs. 31 $40 \%), 0-9 \%$ vs. $31-40 \%$ (**** $\mathrm{p}=0.0002$, HR 2.788 , CI 1.614 to 4.817), $0-9 \%$ vs. $>40 \%$ (*** $<<0.0001$, HR 2.869 , CI 1.787 to 4.608), and $10-20 \%$ vs. $>40 \%$ (" $\mathrm{p}=0.0189$, HR 2.109, CI 1.131 to 3.933). For OS, Kaplan-Meier curves comparison demonstrated significant differences between: $0-9 \%$ vs. 10 $20 \%$ (" $\mathrm{p}=0.0239$, HR 1.636, CI1.067 to 2.509$), 0-9 \%$ vs. $21-$ $30 \%{ }^{* * * *} \mathrm{p}=0.0003$, HR 2.638, CI 1.569 to 4.435$), 0-9 \%$ vs. $31-40 \%{ }^{(* * *} \mathrm{p}=0.024$, HR 2.252, CI1.332 to 3.805), and 0-9\% vs. $>40 \%$ ( ${ }^{* * * *} \mathrm{p}<0.0001$, HR 2.478 , CI 1.565 to 3.922$)$. Since PFS and OS were not significantly different in $21-30 \%$, 31$40 \%$, and $>40 \%$, these groups were combined to one group (>20\%). A survival curve comparison indicated a highly significant difference between $0-9 \%$ and > 20\% mean MGMT methylation in terms of PFS and OS. Consequently, we introduced three major methylation groups: unmethylated 0-9\% (UM), low methylated 10-20\% (LM) and highly methylated $>20 \%$ (HM, Figure $1 \mathrm{c}, \mathrm{d}$ ). mPFS was 7.2 months in the UM group, 10.4 months in the LM group and 19.83 months in the HM group. Kaplan-Meier curve comparison revealed significant differences between UM vs. LM (** $\mathrm{p}=0.0046$, HR 2.225, CI 1.280 to 3.869), LM vs. HM (" $\mathrm{p}=0.0104, \mathrm{HR} 4.224, \mathrm{CI} 2.443$ to 7.303$)$, and UM vs. HM ( ${ }^{* * *} \mathrm{p}<0.0001$, HR 2.439, CI 1.233 to 4.826$)$. mOS was 13.4 months in the UM group vs. 17.9 months in the LM group vs. 29.93 months in the HM group. Survival differences were not significant for UM vs. LM ( $\mathrm{p}=$ NS, HR 1.619, CI 0.9780 to 2.680) and for LM vs. HM ( $\mathrm{p}=$ NS, HR 1.619, CI 0.9780 to 2.680), which was due to one LTS patients within the LM group. OS was significantly different between UM vs. HM (**** $\mathrm{p}<0.0001$, HR 2.900, CI 1.816 to 4.630$)$.

\section{Defining a transition zone}

LM patients demonstrated a similar clinical course compared to UM patients in terms of PFS and OS, which indicated that the widely used PSQ cutoff of $10 \%$ does not fully reflect the clinical response to alkylating agents. We have therefore defined the LM group (10-20\%) as a "transition zone" between unmethylated and clearly methylated cases. To validate the PSQ MGMT results in this particular subgroup of the unselected study cohort, these cases $(L M, n=35)$ were additionally analyzed by sqMSP $(n=32 / 35)$. In $53.1 \%(n=17 / 32)$ sqMSP and PSQ results were disconcordant (representative MSP and PSQ results are shown in Figures 2 c, d). For $n=22 / 35$ 

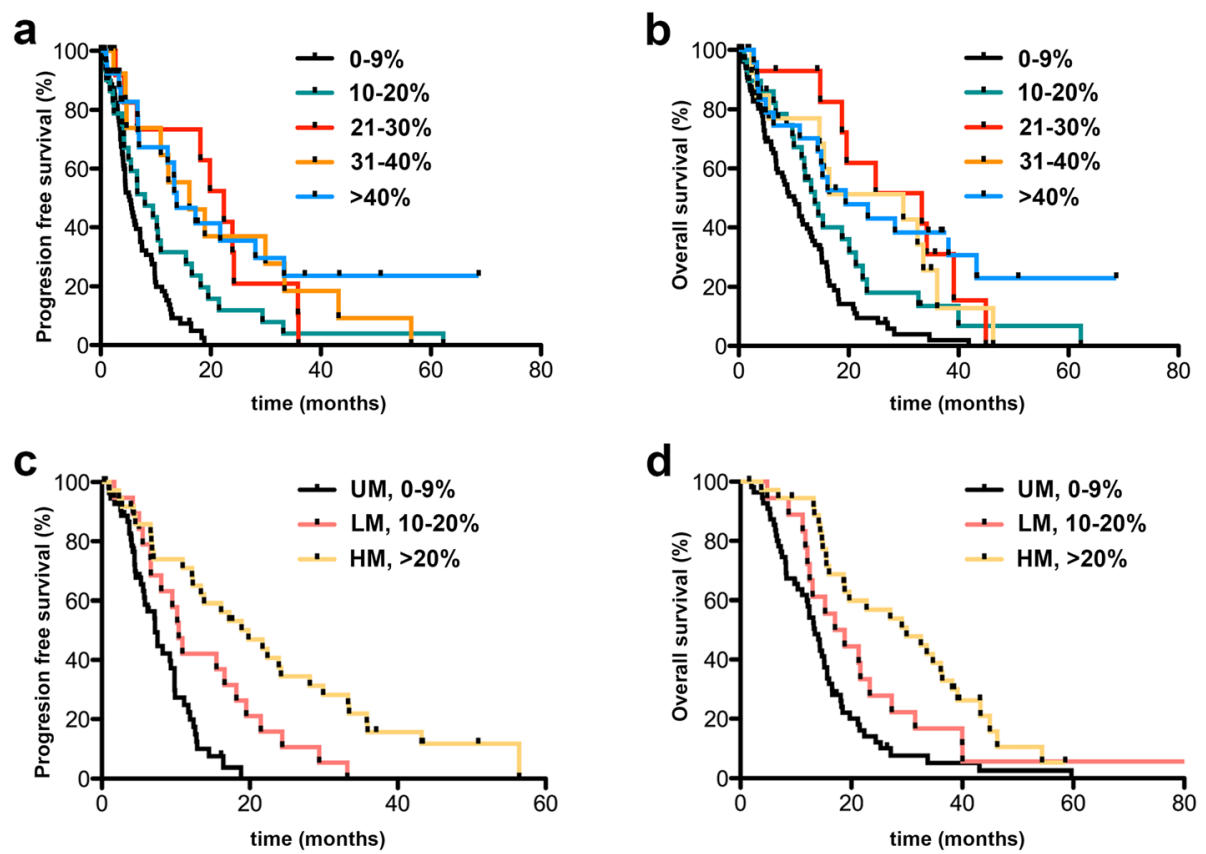

Fig. 1 a, b: Kaplan-Meier curves for progression-free (PFS) and overall survival (OS) of subgroup analysis comparing the different methylation groups (mean MGMT promoter methylation): 0-9\%, 10-20\%, 21-30\%, 31-40\%, and > 40\%. c, d: Kaplan-Meier curves for progression-free (PFS) and overall survival (OS) of subgroup analysis comparing the different methylation groups UM, LM, and HM according to mean MGMT methylation PSQ results

cases, additional dBiseq data was available (representative results in Fig. 2e). PSQ and dBiseq showed identical results in only $45.5 \%(\mathrm{n}=10 / 22)$, MSP and dBiseq in $90 \%$ $(\mathrm{n}=18 / 20)$ of cases. The detailed results are displayed in Additional file 1: Table S1. In general, in cases with PSQ $\geq 16 \%$, we observed a very high consistency between PSQ, MSP and dBiseq results.

We additionally investigated the survival profiles of all transition zone patients after combining PSQ and MSP results. First, we redistributed the LM patients to either the UM or HM category based on MSP testing. As expected, the differences between UM vs. HM were highly significant: PFS $(* * * \mathrm{p}<0.0001, \mathrm{HR} 3.002$, CI 1.886 to 4.778) and OS ( ${ }^{* * * *} \mathrm{p}<0.0001$, HR 2.629, CI 1.729 to 3.997, Additional file 1: Figure S6 a, b). Next, we defined the following more detailed four groups to investigate if the integration of MSP resulted in a redistribution of LM patients to either the UM or HM category: UM, LM + MSP unmethylated, LM + MSP methylated, and HM. The results still clearly indicated a transition zone for median PFS and OS, which seemed independent of the MSP results (Additional file 1: Figure S6 c, d). Moreover, curve comparison between PSQ LM + MSP unmethylated and PSQ LM + MSP methylated showed no significant difference, most likely due to small sample size and presence of one LTS patients within the LM group.

Regarding the aforementioned results, we performed ROC curve analysis for prognostic test evaluation for
PSQ (cutoff 10\%) alone and for PSQ (cutoff 10\%) combined with sqMSP results. LM cases that were considered MGMT unmethylated by sqMSP were therefore assigned to the UM group, LM cases that were considered MGMT methylated by sqMSP were therefore assigned to the HM group. ROC curve analysis revealed superior test precision with an AUC $=0.76$ for PSQ (cutoff 10\%) combined with sqMSP results compared to PSQ (cutoff 10\%) alone (AUC = 0.67; Figure 2 a). Additionally, we performed step-wise cutoff testing for $10 \%$, $12 \%, 15 \%, 17 \%$, and $20 \%$ PSQ results. At a cutoff of $17 \%$, highest test precision was reached with an AUC of 0.77 (Figure $2 \mathrm{~b}$ ).

\section{Discussion}

We demonstrate that $I D H$ wildtype GBM patients with low methylation of the MGMT promoter (mean 10-20\%) represent a "transition zone" in terms of PFS and OS compared to clearly unmethylated (0-9\%) and highly methylated $(>20 \%)$ patients. For patients with low methylated MGMT promoter (10-20\%), PSQ results could be validated in only $51.5 \%(\mathrm{n}=17 / 33$ samples, Additional file 1 : Table S1) by one other method (sqMSP or dBiseq) to be clearly methylated.

Both, MSP and PSQ, have independently been suggested as the "gold standard" for methylation analysis of the $M G M T$ gene promoter $[3,11]$. As to which method to use, the scientific community has not reached a consensus yet 


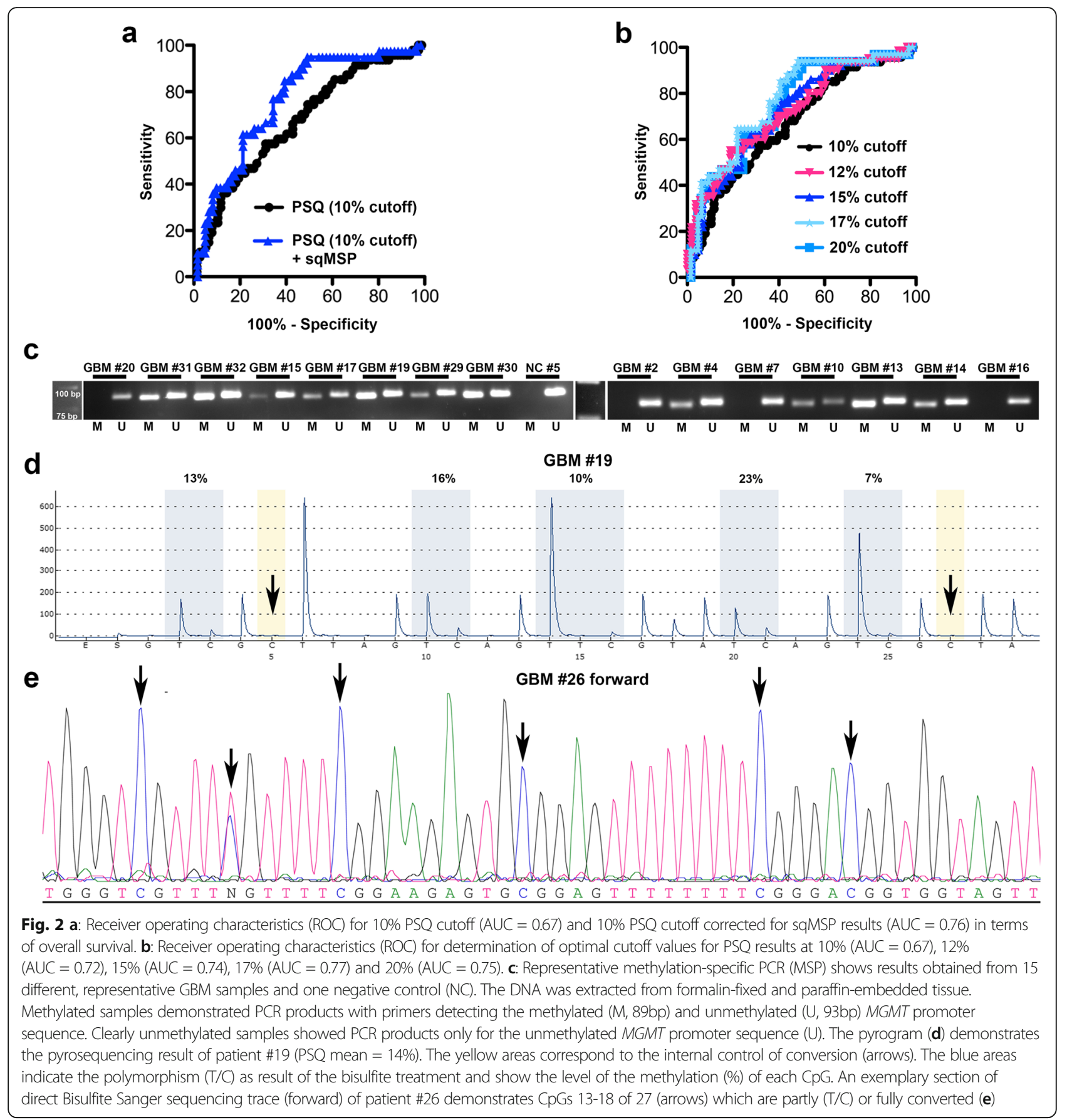

$[3,19]$. Several studies have demonstrated the prognostic value of MSP. Nevertheless, MSP primers are generated to detect either unmethylated or fully methylated MGMT promoter sites, which may in turn result in a lower sensitivity of this method [10]. Furthermore, MSP lacks international standardization [19]. In contrast to MSP, PSQ provides information about the extent of methylation at each individual CpG site, which improves the sensitivity of analyzing heterogeneous methylation patterns within a tumor sample
[10]. Nevertheless, the optimal cutoff value is still a matter of scientific debate [1]. The predictive cutoff is strongly influenced by i) interlaboratory differences, ii) technical challenges of MGMT testing, which are strongly dependent on successful bisulfite treatment of the DNA [6], and particularly iii) tissue processing, such as formalin-fixation and paraffin-embedding $[17,18]$. Therefore, determining a "grey zone" seems to be a more reasonable approach than setting a very strict cutoff. 
Even though previous studies have identified $10 \%$ as the PSQ cutoff to distinguish methylated from unmethylated samples - often based on biological determinants comparing non-neoplastic to neoplastic tissue $[4,17,27$, 28] - several more recent studies have suggested to introduce a "transition" or "grey zone" [7, 17, 18, 26] for partly methylated tumors that perhaps cannot be assigned to either the methylated or unmethylated category. Many of these studies were criticized due to small sample size and heterogeneous patient population [28] including different therapeutic regimens and $I D H \mathrm{mu}-$ tant as well as $I D H$ wildtype GBM patients.

Seeing that $I D H$ mutant GBMs demonstrate a hypermethylator phenotype and show a favorable clinical course, the impact of MGMT methylation on survival may have been overestimated in those studies [23].

Clearly, our study also has some limitations that restrict the interpretation of our data. There are both, the retrospective character and the single center experience. Nevertheless, a key advantage of this study is that it provides a large data set $(n=111)$ from a both clinically and molecularly very well-documented and characterized subgroup of $I D H$ wildtype GBM patients (according to the most recent WHO classification).

As the different methylation groups demonstrate a very distinct clinical course in terms of PFS and OS, and PSQ and sqMSP/sBiseq results are only concordant in $51.5 \%$ of LM patients - which might partly be explained by a heterogeneous methylation pattern and techniquedependent analysis of different CpG sites within the MGMT promoter [19] - we conclude that PSQ results in patients with low MGMT promoter methylation (10$20 \%)$ should be interpreted with caution. If therapeutically relevant, a second technique, e.g. MSP could be additionally used to substantiate the results in MGMT PSQ transitional (10-20\%) cases. Our ROC curve analysis indicates that the combination of PSQ and MSP results is diagnostically beneficial in the LM patient cohort. Our results, furthermore, suggest $17 \%$ as the most accurate cutoff value for PSQ analysis. It has been the consensus in clinical practice to also treat patients with low level MGMT methylation as a potential benefit cannot be excluded. Nevertheless, further scientific investigation is necessary to establish this efficacy. Especially in elderly ( $\geq 70$ years) or fragile GBM patients, a further stratification would be favorable as these patients have a higher risk of chemotherapy-related toxicity and demonstrate less survival benefit from alkylating agents if MGMT is unmethylated [19]. To conclude, we recommend the following classification system be used (particularly if FFPE samples are used): clearly unmethylated $(<10 \%)$, low methylated (between 10-20\%), and clearly methylated (> 20\%), which correlated with significantly improved PFS and OS in our cohort.

\section{Additional file}

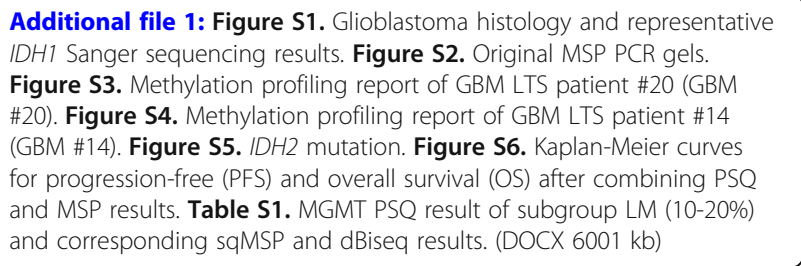

\section{Abbreviations}

CCNU: cyclonexyl-chloroethyl-nitrosourea; dBiSeq: direct bisulfite sequencing; FFPE: formalin-fixed and paraffin embedded; GTR: gross total resection; H\&E: hematoxylin and eosin; HM: highly methylated; IDH: isocitrate dehydrogenase; LM: low methylated; meth: methylated; MGMT: O(6)Methylguanine-DNA methyltransferase; OS: overall survival; PFS: progressionfree survival; PSQ: Pyrosequencing; ROC: receiver operating characteristic; sqMSP: semi-quantitative methylation specific PCR; TMZ: Temozolomide; UM: unmethylated; unmeth: unmethylated

\section{Acknowledgements}

The authors thank Petra Matylewski for excellent technical assistance. JR is a participant of the BIH-Charité Clinical Scientist Program funded by the Charité - Universitätsmedizin Berlin and the Berlin Institute of Health. The authors gratefully thank Hans-Hilmar Goebel and Lara Fleck for critically reading and editing the manuscript.

\section{Authors' contributions}

$\mathrm{JR}$, AK and JO designed the study concept, performed data analysis, participated in data acquisition and wrote the manuscript. RK, ES, FP, and CH performed data analysis, participated in data acquisition, and revised the manuscript. KL performed the statistical data analysis and revised the manuscript. FP, MM, FL, FLH, and PV revised the manuscript and contributed to data analysis. All authors read and approved the final manuscript.

\section{Funding}

This work was supported by the German Cancer Consortium (DKTK), Heidelberg, Germany, Partner Site Charité Berlin, Berlin, Germany. We acknowledge support from the German Research Foundation (DFG) and the Open Access Publication Fund of Charité - Universitätsmedizin Berlin.

\section{Availability of data and materials}

The datasets supporting the conclusions of this article are included within the article and its additional file.

\section{Ethics approval and consent to participate}

All procedures performed in studies involving human participants were in accordance with the ethical standards of the institutional research committee and with the 1964 Helsinki declaration and its later amendments or comparable ethical standards.

\section{Competing interests}

The authors declare that they have no competing interests.

\section{Author details}

${ }^{1}$ Department of Neuropathology, Charité - Universitätsmedizin Berlin, corporate member of Freie Universität Berlin, Humboldt-Universität zu Berlin, and Berlin Institute of Health, Charitéplatz 1 (Virchowweg 15), 10117 Berlin, Germany. ${ }^{2}$ German Cancer Consortium (DKTK), Heidelberg, Germany, Partner Site Charité Berlin, Berlin, Germany. ㄹepartment of Neurosurgery, Charité Universitätsmedizin Berlin, corporate member of Freie Universität Berlin, Humboldt-Universität zu Berlin, and Berlin Institute of Health, Charitéplatz 1, 10117 Berlin, Germany. ${ }^{4}$ Charité - Universitätsmedizin Berlin, corporate member of Freie Universität Berlin, Humboldt-Universität zu Berlin, and Berlin Institute of Health, Institute of Biometrics and Clinical Epidemiology, Berlin, Germany. ${ }^{5}$ Cluster of Excellence, NeuroCure, Charitéplatz 1, 10117 Berlin, Germany. ${ }^{6} \mathrm{BSIO}$ Berlin School of Integrative Oncology, University Medicine Charité, 13353 Berlin, Germany. ${ }^{7}$ Berlin Institute of Health (BIH), 10178 Berlin, 
Germany. ${ }^{8}$ German Center for Neurodegenerative Diseases (DZNE) Berlin, 10117 Berlin, Germany.

\section{Received: 22 April 2019 Accepted: 20 May 2019} Published online: 05 June 2019

\section{References}

1. Bienkowski M, Berghoff AS, Marosi C, Wohrer A, Heinzl H, Hainfellner JA, Preusser M (2015) Clinical Neuropathology practice guide 5-2015: MGMT methylation pyrosequencing in glioblastoma: unresolved issues and open questions. Clin Neuropathol 34:250-257

2. Capper D, Stichel D, Sahm F, Jones DTW, Schrimpf D, Sill M, Schmid S, Hovestadt V, Reuss DE, Koelsche C, Reinhardt A, Wefers AK, Huang K, Sievers P, Ebrahimi A, Scholer A, Teichmann D, Koch A, Hanggi D, Unterberg A, Platten M, Wick W, Witt O, Milde T, Korshunov A, Pfister SM, von Deimling A (2018) Practical implementation of DNA methylation and copy-numberbased CNS tumor diagnostics: the Heidelberg experience. Acta Neuropathol 136:181-210. https://doi.org/10.1007/s00401-018-1879-y

3. Christians A, Hartmann C, Benner A, Meyer J, von Deimling A, Weller M, Wick W, Weiler M (2012) Prognostic value of three different methods of MGMT promoter methylation analysis in a prospective trial on newly diagnosed glioblastoma. PLoS One 7:e33449. https://doi.org/10.1371/journal. pone.0033449

4. Dunn J, Baborie A, Alam F, Joyce K, Moxham M, Sibson R, Crooks D, Husband D, Shenoy A, Brodbelt A, Wong H, Liloglou T, Haylock B, Walker C (2009) Extent of MGMT promoter methylation correlates with outcome in glioblastomas given temozolomide and radiotherapy. Br J Cancer 101:124131. https://doi.org/10.1038/sj.bjc.6605127

5. Felsberg J, Rapp M, Loeser S, Fimmers R, Stummer W, Goeppert M, Steiger HJ, Friedensdorf B, Reifenberger G, Sabel MC (2009) Prognostic significance of molecular markers and extent of resection in primary glioblastoma patients. Clinical cancer research: an official journal of the American Association for Cancer Research 15:6683-6693. https://doi.org/10.1158/10780432.CCR-08-2801

6. Genereux DP, Johnson WC, Burden AF, Stoger R, Laird CD (2008) Errors in the bisulfite conversion of DNA: modulating inappropriate- and failedconversion frequencies. Nucleic Acids Res 36:e150. https://doi.org/10.1093/ nar/gkn691

7. Hegi ME, Genbrugge E, Gorlia T, Stupp R, Gilbert MR, Chinot OL, Nabors LB, Jones G, van Criekinge W, Straub J, Weller M (2018) MGMT Promoter Methylation Cutoff with Safety Margin for Selecting Glioblastoma Patients into Trials Omitting Temozolomide. A Pooled Analysis of Four Clinical Trials. Clinical cancer research : an official journal of the American Association for Cancer Research. doi:https://doi.org/10.1158/1078-0432.CCR-18-3181

8. Herrlinger U, Tzaridis T, Mack F, Steinbach J, Schlegel U, Sabel M, Hau P, Kortman R-D, Krex D, Grauer O, Goldbrunner R, Schnell O, Baehr O, Uhl M, Tabatabai G, Ringel F, Schmidt-Graf F, Brehmer S, Weyerbrock A, Bullinger L, Vajkoczy P, Vatter H, Schäfer N, Kebir S, Weller J, Stummer W, Simon M, Keil V, Nelles M, Fimmers R, Pietsch T, Hattingen E, Coch C, Glas M (2017) ACTR58. PHASE III TRIAL OF CCNU/TEMOZOLOMIDE (TMZ) COMBINATION THERAPY VS. STANDARD TMZ THERAPY FOR NEWLY DIAGNOSED MGMTMETHYLATED GLIOBLASTOMA PATIENTS: THE CeTeg/NOA-09 trial. Neurooncology 19:vi13-vi14. https://doi.org/10.1093/neuonc/nox168.049

9. Jiang M, Zhang Y, Fei J, Chang X, Fan W, Qian X, Zhang T, Lu D (2010) Rapid quantification of DNA methylation by measuring relative peak heights in direct bisulfite-PCR sequencing traces. Laboratory investigation; a journal of technical methods and pathology 90:282-290. https://doi.org/10. 1038/labinvest.2009.132

10. Johannessen LE, Brandal P, Myklebust TA, Heim S, Micci F, Panagopoulos I (2018) MGMT Gene Promoter Methylation Status - Assessment of Two Pyrosequencing Kits and Three Methylation-specific PCR Methods for their Predictive Capacity in Glioblastomas. Cancer Genomics Proteomics 15:437446. https://doi.org/10.21873/cgp.20102

11. Karayan-Tapon L, Quillien V, Guilhot J, Wager M, Fromont G, Saikali S, Etcheverry A, Hamlat A, Loussouarn D, Campion L, Campone M, Vallette FM, Gratas-RabbiaRe C (2010) Prognostic value of O6-methylguanine-DNA methyltransferase status in glioblastoma patients, assessed by five different methods. J NeuroOncol 97:311-322. https://doi.org/10.1007/s1 1060-009-0031-1

12. Kristensen LS, Michaelsen SR, Dyrbye H, Aslan D, Grunnet K, Christensen IJ, Poulsen HS, Gronbaek K, Broholm H (2016) Assessment of Quantitative and
Allelic MGMT Methylation Patterns as a Prognostic Marker in Glioblastoma. J Neuropathol Exp Neurol 75:246-255. https://doi.org/10.1093/jnen/nlv024

13. Louis DN, Perry A, Reifenberger $G$, von Deimling A, Figarella-Branger $D$, Cavenee WK, Ohgaki H, Wiestler OD, Kleihues P, Ellison DW (2016) The 2016 World Health Organization Classification of Tumors of the Central Nervous System: a summary. Acta Neuropathol 131:803-820. https://doi.org/10.1007/ s00401-016-1545-1

14. Mikeska T, Bock C, El-Maarri O, Hubner A, Ehrentraut D, Schramm J, Felsberg J, Kahl P, Buttner R, Pietsch T, Waha A (2007) Optimization of quantitative MGMT promoter methylation analysis using pyrosequencing and combined bisulfite restriction analysis. J Mol Diagn 9:368-381. https://doi.org/10.2353/ jmoldx.2007.060167

15. Parker NR, Hudson AL, Khong P, Parkinson JF, Dwight T, Ikin RJ, Zhu Y, Cheng ZJ, Vafaee F, Chen J, Wheeler HR, Howell VM (2016) Intratumoral heterogeneity identified at the epigenetic, genetic and transcriptional level in glioblastoma. Sci Rep 6:22477. https://doi.org/10.1038/srep22477

16. Parrish RR, Day JJ, Lubin FD (2012) Direct bisulfite sequencing for examination of DNA methylation with gene and nucleotide resolution from brain tissues. Curr Protoc Neurosci Chapter 7:Unit 7 24. https://doi.org/10. 1002/0471142301.ns0724s60

17. Quillien V, Lavenu A, Ducray F, Joly MO, Chinot O, Fina F, Sanson M, Carpentier C, Karayan-Tapon L, Rivet P, Entz-Werle N, Legrain M, Zalcman EL, Levallet G, Escande F, Ramirez C, Chiforeanu D, Vauleon E, Figarella-Branger $D$ (2016) Validation of the high-performance of pyrosequencing for clinical MGMT testing on a cohort of glioblastoma patients from a prospective dedicated multicentric trial. Oncotarget 7:61916-61929. https://doi.org/10. 18632/oncotarget.11322

18. Quillien V, Lavenu A, Sanson M, Legrain M, Dubus P, Karayan-Tapon L, Mosser J, Ichimura K, Figarella-Branger D (2014) Outcome-based determination of optimal pyrosequencing assay for MGMT methylation detection in glioblastoma patients. J Neuro-Oncol 116:487-496. https://doi. org/10.1007/s1 1060-013-1332-y

19. Reifenberger $G$, Hentschel B, Felsberg J, Schackert G, Simon M, Schnell O, Westphal M, Wick W, Pietsch T, Loeffler M, Weller M, German Glioma N (2012) Predictive impact of MGMT promoter methylation in glioblastoma of the elderly. Int J Cancer 131:1342-1350. https://doi.org/10.1002/ijc.27385

20. Stupp R, Hegi ME, Mason WP, van den Bent MJ, Taphoorn MJ, Janzer RC, Ludwin SK, Allgeier A, Fisher B, Belanger K, Hau P, Brandes AA, Gijtenbeek J, Marosi C, Vecht CJ, Mokhtari K, Wesseling P, Villa S, Eisenhauer E, Gorlia T, Weller M, Lacombe D, Cairncross JG, Mirimanoff RO (2009) Effects of radiotherapy with concomitant and adjuvant temozolomide versus radiotherapy alone on survival in glioblastoma in a randomised phase III study: 5-year analysis of the EORTC-NCIC trial. The Lancet Oncology 10:459466. https://doi.org/10.1016/s1470-2045(09)70025-7

21. Stupp R, Taillibert S, Kanner AA, Kesari S, Steinberg DM, Toms SA, Taylor LP, Lieberman F, Silvani A, Fink KL, Barnett GH, Zhu JJ, Henson JW, Engelhard HH, Chen TC, Tran DD, Sroubek J, Tran ND, Hottinger AF, Landolfi J, Desai R, Caroli M, Kew Y, Honnorat J, Idbaih A, Kirson ED, Weinberg U, Palti Y, Hegi ME, Ram Z (2015) Maintenance Therapy With Tumor-Treating Fields Plus Temozolomide vs Temozolomide Alone for Glioblastoma: A Randomized Clinical Trial. Jama 314:2535-2543. https://doi.org/10.1001/jama.2015.16669

22. Suchorska B, Weller M, Tabatabai G, Senft C, Hau P, Sabel MC, Herrlinger U, Ketter R, Schlegel U, Marosi C, Reifenberger G, Wick W, Tonn JC, Wirsching HG (2016) Complete resection of contrast-enhancing tumor volume is associated with improved survival in recurrent glioblastoma-results from the DIRECTOR trial. Neuro-oncology 18:549-556. https://doi.org/10.1093/ neuonc/nov326

23. Turcan S, Rohle D, Goenka A, Walsh LA, Fang F, Yilmaz E, Campos C, Fabius AW, Lu C, Ward PS, Thompson CB, Kaufman A, Guryanova O, Levine R, Heguy A, Viale A, Morris LG, Huse JT, Mellinghoff IK, Chan TA (2012) IDH1 mutation is sufficient to establish the glioma hypermethylator phenotype. Nature 483:479-483. https://doi.org/10.1038/nature10866

24. Weller M, Tabatabai G, Kastner B, Felsberg J, Steinbach JP, Wick A, Schnell O, Hau P, Herrlinger U, Sabel MC, Wirsching HG, Ketter R, Bahr O, Platten M, Tonn JC, Schlegel U, Marosi C, Goldbrunner R, Stupp R, Homicsko K, Pichler J, Nikkhah G, Meixensberger J, Vajkoczy P, Kollias S, Husing J, Reifenberger G, Wick W, Group DS (2015) MGMT Promoter Methylation Is a Strong Prognostic Biomarker for Benefit from Dose-Intensified Temozolomide Rechallenge in Progressive Glioblastoma: The DIRECTOR Trial. Clinical cancer research: an official journal of the American Association for Cancer Research 21:2057-2064. https://doi.org/10.1158/1078-0432.CCR-14-2737 
25. Wen PY, Macdonald DR, Reardon DA, Cloughesy TF, Sorensen AG, Galanis E, Degroot J, Wick W, Gilbert MR, Lassman AB, Tsien C, Mikkelsen T, Wong ET, Chamberlain MC, Stupp R, Lamborn KR, Vogelbaum MA, van den Bent MJ, Chang SM (2010) Updated response assessment criteria for high-grade gliomas: response assessment in neuro-oncology working group. J Clin Oncol Off J Am Soc Clin Oncol 28:1963-1972. https://doi.org/10.1200/JCO. 2009.26.3541

26. Wick W, Weller M, van den Bent M, Sanson M, Weiler M, von Deimling A, Plass C, Hegi M, Platten M, Reifenberger G (2014) MGMT testing--the challenges for biomarker-based glioma treatment. Nat Rev Neurol 10:372385. https://doi.org/10.1038/nrneurol.2014.100

27. Xie H, Tubbs R, Yang B (2015) Detection of MGMT promoter methylation in glioblastoma using pyrosequencing. Int J Clin Exp Pathol 8:1790-1796

28. Zhao H, Wang S, Song C, Zha Y, Li L (2016) The prognostic value of MGMT promoter status by pyrosequencing assay for glioblastoma patients' survival: a meta-analysis. World journal of surgical oncology 14:261. https://doi.org/ $10.1186 / s 12957-016-1012-4$

\section{Publisher's Note}

Springer Nature remains neutral with regard to jurisdictional claims in published maps and institutional affiliations.

\section{Ready to submit your research? Choose BMC and benefit from:}

- fast, convenient online submission

- thorough peer review by experienced researchers in your field

- rapid publication on acceptance

- support for research data, including large and complex data types

- gold Open Access which fosters wider collaboration and increased citations

- maximum visibility for your research: over $100 \mathrm{M}$ website views per year

At $\mathrm{BMC}$, research is always in progress.

Learn more biomedcentral.com/submissions 